\title{
Impact of assimilable nitrogen availability in glucose uptake kinetics in Saccharomyces cerevisiae during alcoholic fermentation
}

\author{
Margarida Palma $a^{1,2}$, Sara Cordeiro Madeira ${ }^{3,4}$, Ana Mendes-Ferreira ${ }^{5}$ and Isabel Sá-Correia ${ }^{1,2^{*}}$
}

\begin{abstract}
Background: The expression and activity of the different Saccharomyces cerevisiae hexose uptake systems (Hxt) and the kinetics of glucose uptake are considered essential to industrial alcoholic fermentation performance. However, the dynamics of glucose uptake kinetics during the different stages of fermentation, depending on glucose and nitrogen availability, is very poorly characterized. The objective of the present work was to examine thoroughly the alterations occurring in glucose uptake kinetics during alcoholic fermentation, by the wine strain S. cerevisiae PYCC 4072, of a synthetic grape juice basal medium with either a limiting or non-limiting initial nitrogen concentration and following nitrogen supplementation of the nitrogen-depleted sluggish fermentation.

Results: Independently of the initial concentration of the nitrogen source, glucose transport capacity is maximal during the early stages of fermentation and presumably sustained by the low-affinity and high-capacity glucose transporter Hxt1p. During nitrogen-limited sluggish fermentation, glucose uptake capacity was reduced to approximately $20 \%$ of its initial values ( $V_{\max }=4.9 \pm 0.8$ compared to $21.9 \pm 1.2 \mu \mathrm{mol} \mathrm{h}^{-1} 10^{-8}$ cells), being presumably sustained by the low-affinity glucose transporter Hxt3p (considering the calculated $K_{\mathrm{m}}=39.2 \pm 8.6 \mathrm{mM}$ ). The supplementation of the sluggish fermentation broth with ammonium led to the increase of glucose transport capacity associated to the expression of different glucose uptake systems with low and high affinities for glucose $\left(K_{\mathrm{m}}=58.2 \pm 9.1\right.$ and $\left.2.7 \pm 0.4 \mathrm{mM}\right)$. A biclustering analysis carried out using microarray data, previously obtained for this yeast strain transcriptional response to equivalent fermentation conditions, indicates that the activation of the expression of genes encoding the glucose transporters $\mathrm{Hxt} 2 \mathrm{p}$ (during the transition period to active fermentation) and Hxt3p, Hxt4p, Hxt6p and Hxt7p (during the period of active fermentation) may have a major role in the recovery of glucose uptake rate following ammonium supplementation. These results suggest a general derepression of the glucose-repressible HXT genes and are consistent with the downregulation of Mig1p and Rgt1p.

(Continued on next page)
\end{abstract}

\footnotetext{
* Correspondence: isacorreia@ist.utl.pt

${ }^{1}$ Institute for Biotechnology and Bioengineering, Centre for Biological and

Chemical Engineering, Instituto Superior Técnico, Technical University of

Lisbon, 1049-001 Lisbon, Portugal

${ }^{2}$ Department of Bioengineering, Instituto Superior Técnico, Technical

University of Lisbon, 1049-001 Lisbon, Portugal

Full list of author information is available at the end of the article
} 
(Continued from previous page)

Conclusions: Although reduced, glucose uptake rate during nitrogen-limited fermentation is not abrogated. Following ammonium supplementation, sluggish fermentation recovery is associated to the increase of glucose uptake capacity, related to the de novo synthesis of glucose transporters with different affinity for glucose and capacity, presumably of Hxt2p, Hxt3p, Hxt4p, Hxt6p and Hxt7p. This study is a contribution to the understanding of yeast response to different stages of alcoholic fermentation at the level of glucose uptake kinetics, in particular under nitrogen limitation or replenish, which is useful knowledge to guide fermentation practices.

Keywords: Hexose transporters, Glucose uptake kinetics, Alcoholic fermentation, Nitrogen regulation, Yeast, Biclustering analysis

\section{Background}

The accumulation of inhibitory metabolites during alcoholic fermentation (e.g. ethanol, acetic acid, long chain fatty acids, etc.) and nutritional deficiencies are considered the main causes of stuck or sluggish fermentations conducted by Saccharomyces cerevisiae [1-4]. The availability of assimilable nitrogen, whose abundance is variable in the natural substrates used for alcoholic fermentation, influences yeast cells performance during the process. The assimilable nitrogen content in grape juice can range from 60 to $2400 \mathrm{mg} \mathrm{L}^{-1}$ [3]; for levels below $120-140 \mathrm{mg} \mathrm{L}^{-1}$, a nitrogen supplementation, usually as diammonium phosphate, is needed to avoid wine fermentation arrest before the complete fermentation of the high sugar concentrations present [3]. The production of other alcoholic beverages, such as beer and the Brazilian spirit cachaça, or of bioethanol from different substrates can also be improved by media supplementation with an assimilable nitrogen source [5-7]. Indeed, it has been proposed that the major limiting factor of enological fermentation occurring upon nitrogen depletion is the inactivation of sugar transporters associated to protein synthesis arrest and their degradation, even when glucose concentration is still high [8-11].

Sugar transport across $S$. cerevisiae plasma membrane is carried out by a complex and highly regulated system of eighteen hexose permeases, from Hxt1p to Hxt17p and Gal2p. The genes HXT1-HXT4 and HXT6,7 are thought to encode the major Hxt proteins since their individual expression is able to restore growth on glucose in a mutant strain deleted for HXT1-7 [12]. The different hexose transporters are transcriptionally regulated in response to glucose availability. For example, the genes encoding low-affinity glucose transporters are activated at high concentrations of glucose (e.g. HXT1) [13,14], or constitutively expressed in the presence of glucose (e.g. HXT3) [14], while the transcription of genes encoding the high-affinity glucose transporters, Hxt6p and Hxt7p is activated when glucose is scarce in the medium $[15,16]$. HXT2 and HXT4 encode proteins with a moderate/high affinity for glucose $[12,17]$, both induced by low levels of glucose and repressed by high levels of glucose, although $H X T 2$ transcription can also be repressed by a different regulatory mechanism when glucose is absent [14]. HXT5, which codes for a moderate-affinity glucose transporter, is not expressed during yeast growth in rich medium with glucose [18], but is transcriptionally activated under conditions leading to low specific growth rate, such as under nitrogen limitation [19-21]. Under specific conditions, the hexose permeases are marked for degradation via endocytosis and transported for degradation in the vacuole [22-24]. For instance, Hxt6p and Hxt7p are internalized by endocytosis under nitrogen starvation when a fermentable carbon source is present [25].

The activity of hexose uptake systems has been considered the factor that limits alcoholic fermentation activity but the variation of sugar uptake kinetics throughout nitrogen-limited sluggish fermentation and the same replenished fermentation, is poorly characterized. The objective of the present work was to examine thoroughly the alteration occurring in glucose uptake kinetics during alcoholic fermentation by the wine strain S. cerevisiae PYCC 4072 of a synthetic grape juice basal medium with either a limiting or non-limiting initial nitrogen concentration and following nitrogen supplementation of the nitrogen-depleted sluggish fermentation. The three different nitrogen availability conditions tested in the present work are similar to those described before [26]. The reanalysis of the gene transcription profiles obtained for the same yeast strain during fermentations carried out under similar nitrogen supply conditions was also carried out by exploring a biclustering algorithm for identification of groups of genes with similar expression patterns in a time series gene expression data [27]. This biclustering analysis provided clues into the alterations occurring at the expression level of hexose transporters encoding genes and their putative transcription regulators during the various fermentation conditions. In particular, the selected biclusters corresponding to the fermentation period following nitrogen supplementation of the nitrogen-limited sluggish fermentation was on the focus of our analysis. 


\section{Methods}

\section{Yeast strain and fermentation media}

S. cerevisiae wine strain PYCC 4072 [26] was maintained at $-80^{\circ} \mathrm{C}$ in YPD medium, containing yeast-peptonedextrose $\left(20 \mathrm{~g} \mathrm{~L}^{-1}\right.$ glucose, $20 \mathrm{~g} \mathrm{~L}^{-1}$ peptone and and 10 $\mathrm{g} \mathrm{L}^{-1}$ yeast extract) and glycerol $(15 \% \mathrm{v} / \mathrm{v})$. Prior to use, cells from frozen cultures were transferred to fresh YPD agar plates $\left(20 \mathrm{~g} \mathrm{~L}^{-1}\right.$ agar), and grown for $24 \mathrm{~h}$ at $30^{\circ} \mathrm{C}$. The previously described chemically defined basal grape juice medium, mimicking a standard natural must with glucose as the sole carbon source [26] was used with minor alterations, specifically the initial available nitrogen concentration present in the three fermentation media and the use of potassium and sodium tartrate instead of potassium tartrate. The medium used contained (per liter): glucose, $200 \mathrm{~g}$; potassium and sodium tartrate, 5 g; L-malic acid, 3 g; citric acid, 0.2 g; $\mathrm{K}_{2} \mathrm{HPO}_{4}$, 1.14 g; $\mathrm{MgSO}_{4} 7 \mathrm{H}_{2} \mathrm{O}, 1.23 \mathrm{~g} ; \mathrm{CaCl}_{2} 2 \mathrm{H}_{2} \mathrm{O}, 0.44 \mathrm{~g} ; \mathrm{MnCl}_{2}$ $4 \mathrm{H}_{2} \mathrm{O}, 198.2 \mu \mathrm{g} ; \mathrm{ZnCl}_{2}, 135.5 \mu \mathrm{g} ; \mathrm{FeCl}_{2}, 32.0 \mu \mathrm{g} ; \mathrm{CuCl}_{2}$, $13.6 \mu \mathrm{g} ; \mathrm{H}_{3} \mathrm{BO}_{3}, 5.7 \mu \mathrm{g} ; \mathrm{Co}\left(\mathrm{NO}_{3}\right)_{2} \quad 6 \mathrm{H}_{2} \mathrm{O}, 29.1 \mu \mathrm{g}$; $\mathrm{NaMoO}_{4} 2 \mathrm{H}_{2} \mathrm{O}, 24.2 \mu \mathrm{g}$; and $\mathrm{KIO}_{3}, 10.8 \mu \mathrm{g}$; vitamins (myo-inositol, $100 \mathrm{mg}$; pyridoxine $\mathrm{HCl}, 2 \mathrm{mg}$; nicotinic acid, $2 \mathrm{mg}$; calcium pantothenate, $1 \mathrm{mg}$; thiamine $\mathrm{HCl}$, $0.5 \mathrm{mg}$; p-amino benzoic acid, $0.2 \mathrm{mg}$; riboflavin, $0.2 \mathrm{mg}$; biotin, $0.125 \mathrm{mg}$; and folic acid, $0.2 \mathrm{mg}$ ). The medium $\mathrm{pH}$ was adjusted to 3.7 prior to sterile filtration. Nitrogen was supplied as diammonium phosphate (DAP), as the sole nitrogen source, to facilitate the monitoring of nitrogen consumption. For this purpose, a sterile stock concentrated solution of diammonium phosphate, with $\mathrm{pH}$ adjusted to 3.7, was added prior to fermentation or during the process following nitrogen source depletion.

\section{Preparation of the inoculum and fermentation conditions} The preparation of $S$. cerevisiae PYCC 4072 inoculum and the fermentation conditions followed essentially those described previously [26]. The inoculum was prepared by pre-growing the yeast culture overnight in 100$\mathrm{mL}$ shake flasks containing $2 / 3$ of this volume $(66 \mathrm{~mL})$ of synthetic grape juice medium with $320 \mathrm{mg} \mathrm{L}^{-1}$ of assimilable nitrogen (corresponding to $1.5 \mathrm{~g} \mathrm{~L}^{-1}$ of diammonium phosphate $\left.\left(\mathrm{NH}_{4}\right)_{2} \mathrm{HPO}_{4}\right)$, at $25^{\circ} \mathrm{C}$ and $150 \mathrm{rpm}$. This preculture was used to inoculate the main cultures with an initial concentration of the viable population of $10^{6}$ colony forming units (CFU) per milliliter. Fermentations were carried out in $500 \mathrm{~mL}$ Erlenmeyer flasks (120 rpm) equipped with cotton stoppers, keeping the ratio volume of grape juice medium:volume of the flask equal to $2 / 3$, at $20^{\circ} \mathrm{C}$, mimicking vinification conditions. The effect of the initial nitrogen concentration on yeast performance during fermentation was examined using a basal grape juice medium containing an initial assimilable nitrogen concentration of either $90 \mathrm{mg} \mathrm{L}^{-1}$ (424 mg L $\mathrm{m}^{-1}$ of $\left.\left(\mathrm{NH}_{4}\right)_{2} \mathrm{HPO}_{4}\right)$ for the nitrogen-limited fermentation (LF) or $320 \mathrm{mg} \mathrm{L}^{-1}\left(1.5 \mathrm{gL}^{-1}\right.$ of $\left(\mathrm{NH}_{4}\right)$ ${ }_{2} \mathrm{HPO}_{4}$ ) for the complete fermentation (CF). After 72 hours of fermentation of the medium with the limited nitrogen concentration (i. e. approximately 2 days after nitrogen depletion) the sluggish fermentation broth was divided into two $250 \mathrm{~mL}$ Erlenmeyer flasks (keeping the ratio volume of grape juice medium:volume of the flask equal to $2 / 3)$, and assimilable nitrogen $\left(230 \mathrm{mg} \mathrm{L}^{-1}\right.$ as $1.1 \mathrm{gL}^{-1}$ of $\left.\left(\mathrm{NH}_{4}\right)_{2} \mathrm{HPO}_{4}\right)$ was added to one of the fermentation broths (identified as the refed fermentation - RF) while the other (LF) remained as the control.

\section{Fermentation monitoring}

Yeast growth was monitored throughout fermentation by following culture optical density at $600 \mathrm{~nm}$ and by the determination of the concentration of viable cells, based on the number of CFU $\mathrm{mL}^{-1}$ grown onto YPD solid medium, incubated for 3 days at $30^{\circ} \mathrm{C}$, and the progress of alcoholic fermentation by the measurement of glucose, assimilable nitrogen and ethanol concentrations. Culture supernatants were obtained by centrifugation of cell cultures harvested after 0, 6, 24, 48, 72, 80, 96 and $144 \mathrm{~h}$ of fermentation. The ammonium concentration was determined as described before [28] and ethanol and glucose concentrations were determined by highpressure liquid chromatography (HPLC) using an Aminex $\mathrm{HPX} 87 \mathrm{H}$ ion exchange chromatography column, eluted at room temperature with $0.005 \mathrm{M} \mathrm{H}_{2} \mathrm{SO}_{4}$ at a

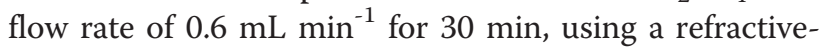
index detector. The retention times for glucose and ethanol were $8.3 \mathrm{~min}$ and $19.4 \mathrm{~min}$, respectively. Reproducibility and linearity of the method were tested, and concentrations were estimated based on appropriate calibration curves. The results represent the average values of three independent experiments. Error bars depict standard deviation from the mean.

\section{Glucose transport assays}

Initial glucose uptake rates were determined at different fermentation times using radiolabeled D-[U- $\left.{ }^{14} \mathrm{C}\right]$ glucose (PerkinElmer, MA, USA, $300 \mathrm{mCi} \mathrm{mmol}^{-1}, 11.1 \mathrm{GBq} \mathrm{mmol}^{-1}$ ) as described previously $[29,30]$ with minor modifications. Cells were harvested by centrifugation from the fermentation broths, washed twice with $10 \mathrm{~mL}$ ice-cold water and resuspended to a density of $10^{9}$ cells $\mathrm{mL}^{-1}$ in TM buffer (0.1 M MES, $41 \mathrm{mM}$ Tris, pH 5.5). Aliquots of $40 \mu \mathrm{L}$ of this cellular suspension were transferred to $5 \mathrm{ml}$ Röhren tubes and incubated at $30^{\circ} \mathrm{C}$ for $5 \mathrm{~min}$ for temperature equilibration. After this period, $10 \mu \mathrm{L}$ of radiolabeled D-[U- $\left.{ }^{14} \mathrm{C}\right]$ glucose was added to each tube by vigorous vortexing. The final concentrations of radiolabeled $\mathrm{D}$-[U- $\left.{ }^{14} \mathrm{C}\right]$-glucose in the tubes were $1,2,5,10,20,50,100$ and $200 \mathrm{mM}$. These radiolabeled glucose solutions were prepared by dilution in distilled water of a $1 \mathrm{M}$ radiolabeled $\mathrm{D}$-[U- $\left.{ }^{14} \mathrm{C}\right]$-glucose 
stock solution. After 5 seconds of cell incubation with the radiolabeled glucose, reaction was stopped by vigorous quenching with $3.5 \mathrm{~mL}$ ice-cold demineralized water. Cells were immediately collected by filtration (Whatman $\mathrm{GF} / \mathrm{C}$ glass microfiber membranes), the filters transferred to scintillation vials containing $7 \mathrm{~mL}$ liquid scintillation cocktail Ultima Gold ${ }^{\mathrm{TM}}$ MV (Perkin-Elmer) and the radioactivity measured in a Beckman LS 5000TD scintillation counter. Sugar uptake rates were calculated using results obtained in duplicate for each sugar concentration and each transport assay was repeated at least twice. The obtained data were fitted to Eadie-Hofstee plots using graphPad Prism (GraphPad Software Inc., La Jolla, CA) to identify the presence of one or two glucose uptake systems. The estimation of the kinetic parameters $K_{\mathrm{m}}$ and $V_{\max }$ was performed using the Michaelis-Menten kinetic model for one or two carriers. An iterative computation procedure described in [31] was used for modeling the two-carrier system using both Michaelis-Menten and Lineweaver-Burk plots.

\section{Biclustering analysis for regulatory module identification}

Results of the transcriptional response of $S$. cerevisiae PYCC 4072 during the three fermentations performed under conditions similar to those examined in the present work [26] were explored using CCC-Biclustering, a state of the art biclustering algorithm specifically developed for time series gene expression data analysis [27]. Biclustering enables the identification of groups of genes with highly coherent expression profiles in a subset of the time points analyzed (local patterns) without forcing all the genes to belong to one group and allowing genes to belong to more than one group. Groups of genes with coherent expression patterns under all the time points analyzed (global patterns) are also discovered, when they exist. Biclustering should thus be preferred to standard clustering techniques, such as those used in [27], in gene expression data analysis. When clustering is used, all genes are forced to belong to one group (cluster), each gene can only belong to one group, and within-group similarities are computed using all the time points analyzed, thus meaning that only global patterns can be identified. This results either in a large number of groups with a short number of genes, which are difficult to analyze and are in most cases uninteresting from a biological point of view, or in a small number of groups with a large number of genes, where some genes behave coherently and the large majority of the remaining genes in the group have uncorrelated expression profiles, as it is the case of most clusters found in [26]. Moreover, clustering algorithms are not able to deal well with missing values, common in expression data, forcing genes with missing values to be deleted or missing value imputation to be performed prior to the clustering analysis, thus augmenting noise. In [26] missing values were filled before clustering.

CCC-Biclustering finds groups of genes with similar expression patterns in contiguous sets of the time points analyzed, thus discovering local expression patterns that would not be uncovered using a global analysis technique, such as clustering. More specifically, and since the algorithm performs an exhaustive search, all maximal CCC-Biclusters (Contiguous Column Coherent Biclusters), maximal sets of genes with maximal expression profiles in consecutive time points, are discovered. In order to avoid the removal of genes with missing expression values at certain time points and therefore guarantee that potentially relevant genes were not excluded a priori from the biclustering analysis, CCC-Biclustering was extended to cope with missing values. The biclusters discovered were then sorted according to the statistical significance ( $p$-value) of their expression pattern [27]. The $p$-value gives the probability of random occurrence of a bicluster (group of genes sharing the same expression profile) in a gene expression matrix of a given size.

The analysis was focused on the biclusters containing the hexose transporter encoding genes, particularly in the period following ammonium supplementation of the nitrogen-limited fermentation.

\section{Results}

Glucose uptake kinetics during alcoholic fermentation under different nitrogen availability conditions

The two different initial nitrogen concentrations present in the fermentation media tested in this study led to either a nitrogen-limited sluggish fermentation (LF) (90 $\mathrm{mg} \mathrm{L}^{-1}$ of assimilable nitrogen) or to a complete fermentation (CF) (320 mg L $\mathrm{m}^{-1}$ of assimilable nitrogen), this one leading to higher final biomass and ethanol concentrations (Figure 1). Even though cell growth stopped in CF medium during the second day of fermentation as the result of nitrogen limitation, all the initial glucose concentration present $\left(200 \mathrm{~g} \mathrm{~L}^{-1}\right)$ was consumed approximately after 3 days of fermentation (Figure 1-C). As the result of nitrogen exhaustion during the first hours of the LF fermentation process, the rate of glucose consumption during the rest of the fermentation became much lower when compared with CF. When this sluggish fermentation broth was supplemented with diammonium phosphate (RF), a period of latency, characterized by a non-proliferating yeast viable cell population, was observed during the first hours that followed the supplementation. When yeast growth and fermentation were actively resumed after this period of latency, biomass production and nitrogen and glucose consumption rates reached values similar to those attained in CF (Figure 1). 

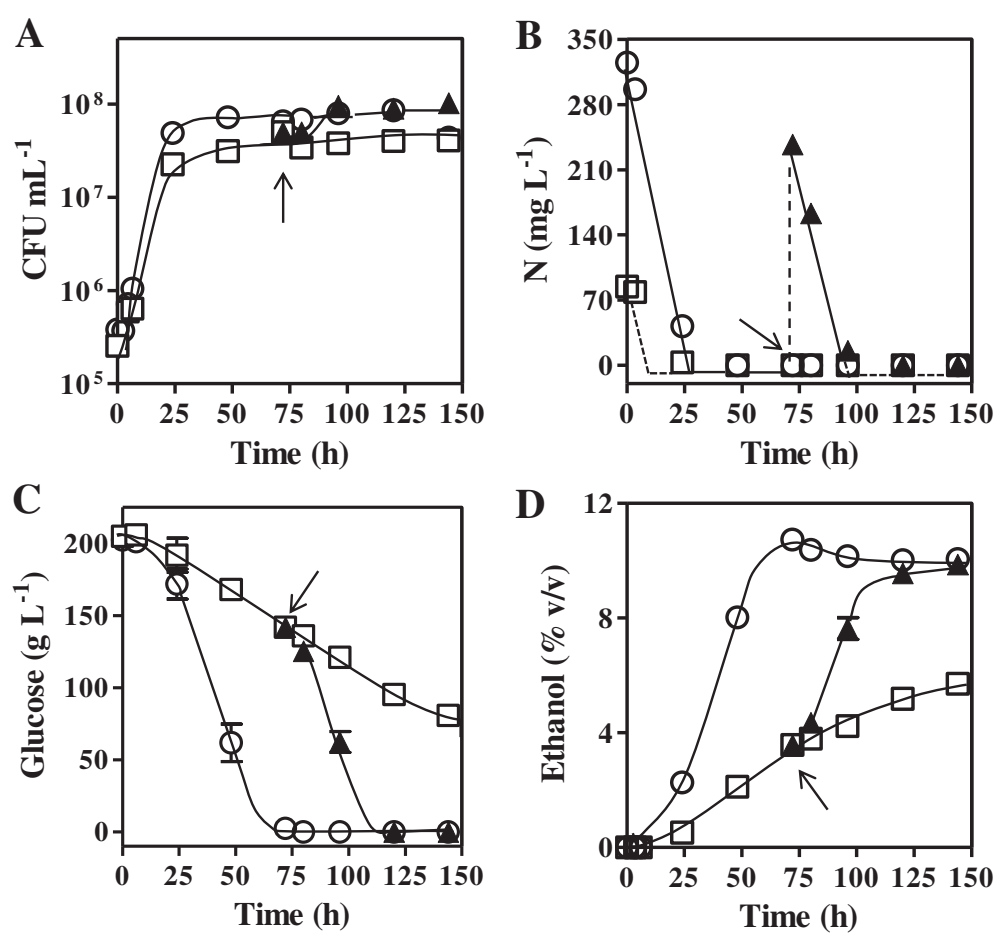

Figure 1 Growth, nutrient consumption and ethanol accumulation during alcoholic fermentation carried out under different nitrogen availability conditions. Growth curves and fermentation performance of S. cerevisiae PYCC 4072 were followed during $144 \mathrm{~h}$ of incubation at $20^{\circ} \mathrm{C}$, by determining the concentrations of: (A) colony forming units (CFU mL ${ }^{-1}$ ), (B) ammonium, (C) glucose and (D) ethanol in the fermentation broth. Yeast cells were cultivated in synthetic basal grape juice medium with $200 \mathrm{~g} \mathrm{~L}^{-1}$ glucose and different nitrogen (N) concentrations leading to: i) complete fermentation (CF) [320 $\left.\mathrm{mg} \mathrm{L}^{-1} \mathrm{~N}\right](\mathrm{O})$; ii) nitrogen-limited fermentation (LF) $\left[90 \mathrm{mg} \mathrm{L}^{-1} \mathrm{~N}\right](\square)$; iii) nitrogen-limited fermentation $\mathrm{LF}$ followed by refed with ammonium after nitrogen exhaustion (RF) $\left[90+230 \mathrm{mg} \mathrm{L}^{-1} \mathrm{~N}\right](\mathbf{\Lambda})$. The arrow indicates the time of nitrogen supplementation in RF.

Moreover, the ethanol concentration attained during CF and RF was about $10 \%(\mathrm{v} / \mathrm{v})$ while in LF only $5 \%(\mathrm{v} / \mathrm{v})$ of ethanol was produced (Figure 1-D).

The values of the kinetic parameters of glucose uptake in yeast cells during the three fermentations under study were determined using radiolabeled D-glucose assays and calculated based on the Eadie-Hofstee and Michaelis-Menten plots (Table 1, Figures 2 and 3). Since these glucose uptake kinetic values represent the contribution of one or several co-expressed transporters, with distinct glucose affinities and uptake capacities $[17,32]$, the subsequent analysis of data took into consideration the referred kinetic parameters of the Hxt1-7p transporters when individually expressed $[17,18,21,32]$. The determination of the kinetic parameters was based on the Michaelis-Menten model that allows, at most, the identification of high- and lowaffinity glucose uptake system components. This means that the eventual presence of a moderate-affinity system would not be detected by this analysis. After $6 \mathrm{~h}$ of fermentation, when the assimilable nitrogen concentration present in both CF and LF conditions did not limit growth, only slight differences in the kinetic parameters of glucose uptake in cells harvested from both fermentation broths were registered (Table 1, Figures 2 and 3). The affinity constant $\left(K_{\mathrm{m}}\right)$ values calculated correspond to a low-affinity glucose uptake system such as Hxt1p $\left(K_{\mathrm{m}}=100 \mathrm{mM}\right)$ [17,32]. As the glucose concentration decreases in CF, a gradual decline in the affinity constant is observed. This decline is less marked in LF and, after $24 \mathrm{~h}$ of fermentation, an Hxt1p-like affinity constant is still observed. A progressive reduction of the transport capacity $\left(V_{\max }\right)$ was registered throughout $\mathrm{CF}$ and LF fermentations until the second day of fermentation. Interestingly, the glucose uptake rate, although significantly reduced during sluggish fermentation (LF), was still detectable (Table 1, Figure 3). Indeed, glucose uptake capacity was not completely abrogated during the extended nitrogen starvation period in LF, remaining approximately $20 \%$ of the initial glucose uptake capacity during process monitoring (Table 1). After glucose depletion from the CF medium (after $72 \mathrm{~h}$ of incubation) it was possible to identify two glucose uptake systems (Table 1, Figure 2), suggesting the presence of both a low-affinity uptake system $\left(K_{\mathrm{m}}=67.5 \pm 14.9 \mathrm{mM}\right.$ or $K_{\mathrm{m}}=45.2 \pm 10.2 \mathrm{mM}$ ) and a high-affinity uptake system 
Table 1 Kinetic parameters $K_{\mathrm{m}}$ and $V_{\max }$ of glucose uptake in yeast cells harvested during the three fermentation curves examined in this study (Figure 1) corresponding to different nitrogen supplementations of the fermentation medium: complete fermentation (CF), nitrogen-limited fermentation (LF) and refed fermentation (RF)

\begin{tabular}{|c|c|c|c|c|c|c|}
\hline \multirow{2}{*}{$\begin{array}{l}\text { Incubation } \\
\text { Time (h) }\end{array}$} & \multicolumn{3}{|l|}{$K_{\mathrm{m}}(\mathrm{mM})$} & \multicolumn{3}{|c|}{ 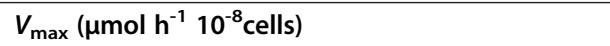 } \\
\hline & $\mathrm{CF}$ & LF & RF & $\mathrm{CF}$ & LF & RF \\
\hline 6 & $99.2 \pm 6.5$ & $108.5 \pm 10.4$ & & $23.2 \pm 0.7$ & $21.9 \pm 1.2$ & \\
\hline 24 & $36.6 \pm 3.5$ & $81.7 \pm 9.4$ & & $13.9 \pm 0.4$ & $11.5 \pm 0.6$ & \\
\hline 48 & $34.2 \pm 4.4$ & $43.8 \pm 5.6$ & & $11.0 \pm 0.5$ & $4.7 \pm 0.2$ & \\
\hline 72 & ND & $31.5 \pm 2.9$ & AS & ND & $4.8 \pm 0.1$ & AS \\
\hline \multirow[t]{2}{*}{80} & $67.5 \pm 14.9$ & $38.9 \pm 6.6$ & $33.2 \pm 3.3$ & $9.1 \pm 0.8$ & $5.1 \pm 0.6$ & $12.9 \pm 0.4$ \\
\hline & $2.1 \pm 0.6$ & & & $3.5 \pm 0.1$ & & \\
\hline \multirow[t]{2}{*}{96} & $45.2 \pm 10.2$ & $39.2 \pm 8.6$ & $58.2 \pm 9.1$ & $8.6 \pm 0.7$ & $4.9 \pm 0.8$ & $8.7 \pm 0.8$ \\
\hline & $1.9 \pm 0.3$ & & $2.7 \pm 0.4$ & $3.8 \pm 0.2$ & & $1.0 \pm 0.1$ \\
\hline \multirow[t]{2}{*}{144} & ND & ND & $33.1 \pm 3.5$ & ND & ND & $7.3 \pm 0.4$ \\
\hline & & & $1.3 \pm 0.3$ & & & $1.3 \pm 0.2$ \\
\hline
\end{tabular}

ND: Not determined.

AS: Ammonium supplementation.

$\left(K_{\mathrm{m}}=2.1 \pm 0.6 \mathrm{mM}\right.$ or $\left.K_{\mathrm{m}}=1.9 \pm 0.3 \mathrm{mM}\right)$ after 80 or $96 \mathrm{~h}$ of fermentation, respectively. The supplementation of LF with ammonium following nitrogen exhaustion resulted in the stimulation of glucose consumption rates (Figure 1C), consistent with the registered increase of glucose uptake capacity eight hours following ammonium refed. During this period, the affinity for glucose, characteristic of a low-affinity uptake system, maintained essentially the values registered in LF cells before ammonium supplementation. The low-affinity glucose transporter was detected during RF, similarly to CF, but contrarily to CF the presence of the high-affinity glucose uptake system in yeast cells from RF was detected even when glucoserepressible concentrations were still present in the fermenting medium $\left(K_{\mathrm{m}}=2.7 \pm 0.4 \mathrm{mM}\right.$, for cells harvested after $96 \mathrm{~h}$ of fermentation) (Table 1, Figure 3).
Biclustering analysis of the transcription profiles of yeast cells following ammonium supplementation of a sluggish fermentation

In order to get clues into the Hxt transporters that may be responsible for glucose uptake in the recovery of glucose uptake rates following ammonium supplementation of LF, a biclustering analysis was applied to the microarray data from the transcriptional response of $S$. cerevisiae PYCC 4072 cultivated under different nitrogen availability conditions similar to those examined in our study [26]. The biclusters containing the hexose transporter encoding genes HXT1-7 are shown in Figure 4. The full list of the genes belonging to each of the selected biclusters is available in Additional file 1. Results from the biclustering analysis carried out indicate that HXT2 was the sole glucose transporter encoding gene found to

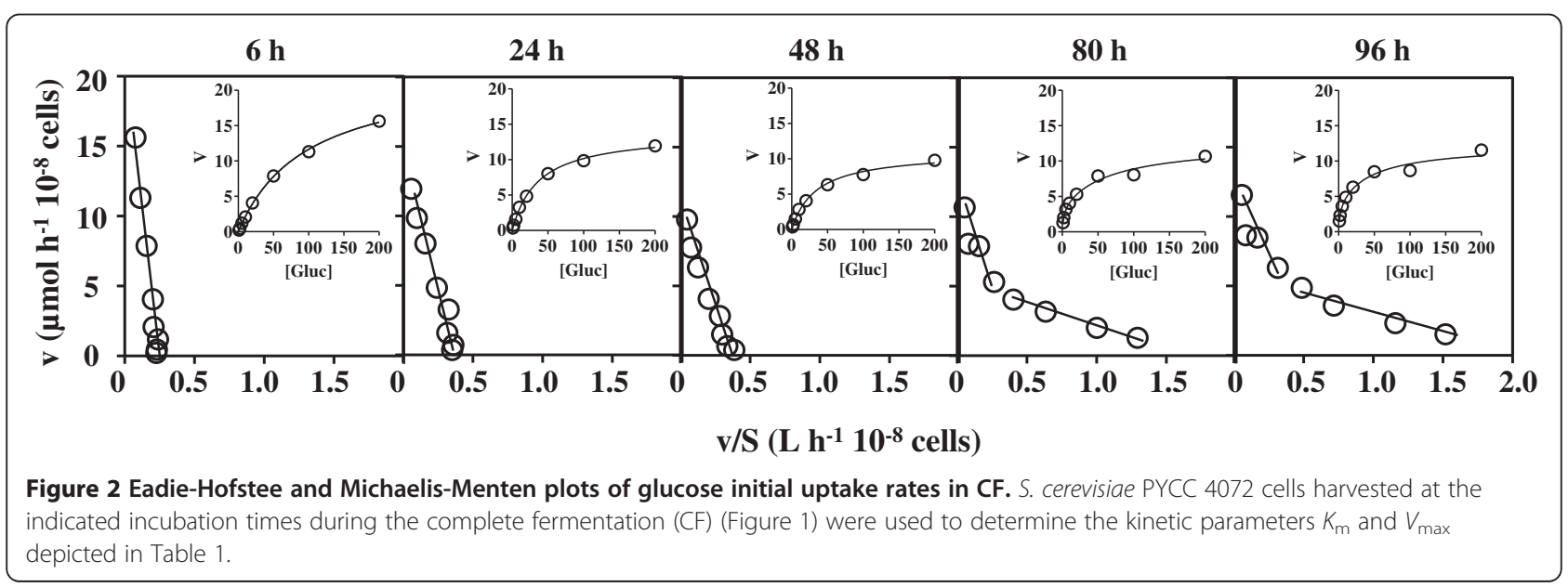



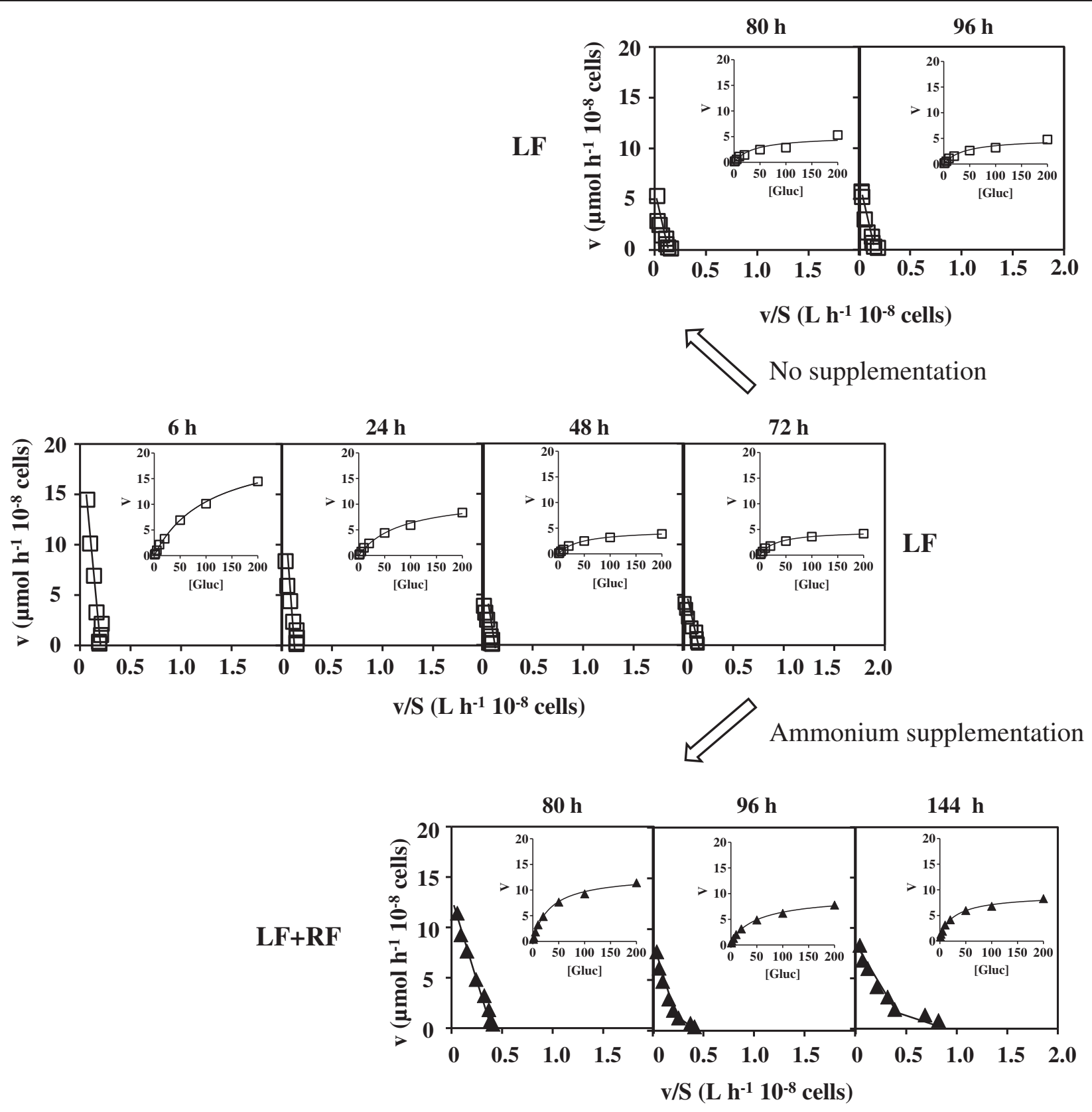

Figure 3 Eadie-Hofstee and Michaelis-Menten plots of glucose initial uptake rates in LF and RF. S. cerevisiae PYCC 4072 cells harvested at the indicated incubation times from the nitrogen-limited fermentation (LF) (Figure 1) that was either or not supplemented with diammonium phosphate 72 hours after fermentation start (LF+RF), were used to determine the kinetic parameters $K_{m}$ and $V_{\max }$ shown in Table 1.

be upregulated during the period of growth latency observed up to $8 \mathrm{~h}$ of incubation following ammonium refed (Figure 4-A, period 1). Since during this period, glucose concentration was above $100 \mathrm{gL}^{-1}$ the increase of HXT2 transcription was not expected based on previous reports on the repression of this gene transcription by Mig1p and Rgt1p under glucose repressing concentrations [14]. However, consistent with this observation, the transcription of MIG1 and RGT1 genes was found to be downregulated during the same period of time (Figure 4-C). Moreover, HXT2 was also previously found to be induced during the small lag phase characteristic of the start of alcoholic fermentation and associated to growth resumption [33]. The transcription level of the genes coding for Hxt3p, Hxt4p, Hxt6p and Hxt7p remained unchanged during the early response to ammonium supplementation, but exhibited a significant increase during the period of 

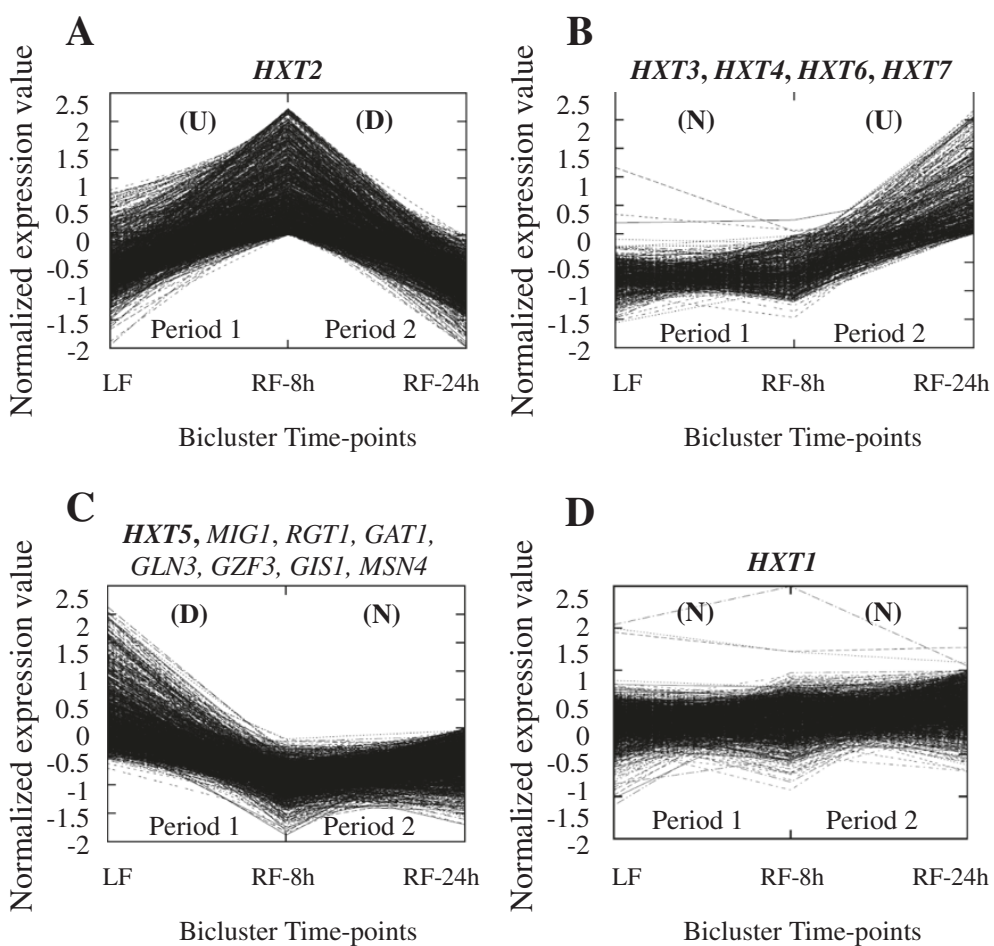

Figure 4 Patterns of the CCC-Biclusters describing the transcriptional changes occurring in hexose transporter genes (in bold) and selected transcription factors following ammonium supplementation (RF) to the nitrogen-limited fermentation (LF). Two incubation time periods were compared following $80 \mathrm{~h}$ of LF fermentation (a control time point): i) after $8 \mathrm{~h}$ of incubation following ammonium supplementation (period 1) and ii) after a period of incubation extending from 8 to $24 \mathrm{~h}$ following ammonium supplementation (period 2). The transcriptional alterations during the selected periods are shown under parenthesis as U (Upregulation), D (Downregulation) and $\mathrm{N}$ (№ changes registered). The full list of genes present in each bicluster is provided in Additional file 1.

intense glycolytic and fermentative activities following growth resumption in the ammonium supplemented broth (Figure 4-B, period 2). This evidence is in agreement with the identification of a glucose uptake kinetics composed of both a low- and a high-affinity glucose uptake system during the same period of fermentation. However, similarly to $H X T 2$, the increase of HXT4, HXT6 and HXT7 transcription levels when glucose-repressible concentrations were present in the external medium was not expected $[14,15]$, suggesting a general derepression of these glucose-repressible HXT genes during this phase. Only HXT5, coding for a moderate-affinity glucose transporter, was found to be downregulated following the addition of ammonium to the sluggish fermentation (Figure 4-C, period 1). Moreover, an Hxt5p-like kinetics could not be detected during the nitrogen-starved fermentation. Based on the information gathered in the YEASTRACT database dedicated to potential regulatory associations between transcription factors and genes [34], which considers both the documented evidences and the presence of binding sites in the promoter sequences of a particular gene, it was possible to identify transcription factors putatively involved in HXT5 transcription, as suggested by the transcription profile similar to the one for HXT5. These include the nitrogen catabolite repression regulators Gat1p, Gln3p and Gzf3p [35], Gis1p, involved in the upregulation of several genes upon nutrient starvation [36] and Msn4p, involved in activation of stress responsive genes [37]. This association is consistent with the reported activation of HXT5 transcription during nitrogen starvation [21] and the presence of STRE and HAP elements in HXT5 promoter regions [38]. The transcription level of the low-affinity glucose transporter encoding gene HXT1 was not altered in response to the addition of ammonium (Figure 4-D) and an Hxt1p-like kinetics for glucose uptake in yeast cells following ammonium supplementation was not either detected.

\section{Discussion}

The kinetics of glucose uptake and the expression and activity of the different hexose uptake systems of $S$. cerevisiae are considered essential to industrial alcoholic fermentation performance. However, the dynamics of glucose uptake kinetics during the different stages of 
fermentation, depending on glucose and nitrogen availability, is very poorly characterized. During the present study, it was shown that glucose transport capacity is maximal during the early stages of fermentation, when nitrogen concentration did not limit growth both in CF and LF media. Under such conditions, glucose transport is essentially sustained by the low-affinity and high-capacity glucose transporter Hxt1p, as suggested by the high $K_{\mathrm{m}}$ and $V_{\max }$ values calculated $[17,32]$. It is known that in the presence of high glucose concentrations available at the beginning of fermentation (above $100 \mathrm{~g} \mathrm{~L}^{-1}$ ), HXT1 transcription is induced by the coordinated activity of the Snf3p/ Rgt2p-Rgt1p and HOG pathways [33,39]. As the nitrogen concentrations available became a growth limiting factor and glucose concentrations decreased, the values calculated for the $K_{\mathrm{m}}$ for glucose decreased to values close to those calculated for the low-affinity glucose transporter Hxt3p when individually expressed $\left(K_{\mathrm{m}}=\right.$ 30-60 mM) [17,32]. The absence of an Hxt1p-like uptake kinetics after nitrogen depletion is consistent with the fact that, under nitrogen starvation, the induction of HXT1 transcription by glucose is repressed and the protein is degraded via the TOR pathway, which controls growth and metabolism in response to nutrient availability $[40,41]$. After the addition of ammonium to a nitrogen-limited fermentation containing HXT1-inducible sugar concentrations, the expected glucose uptake kinetics characteristic of Hxt1 was still not observed, consistent with the lack of variation in HXT1 transcription level following nitrogen supplementation. The lowaffinity glucose transporter Hxt3p was reported to remain functional as long as glucose is available in the fermentation medium [14], but, in this study, we found kinetic evidences that Hxt3p is functional even in the absence of glucose, when ethanol concentrations reached maximum values $(10 \%(\mathrm{v} / \mathrm{v}))$ or during sluggish fermentation. Remarkably, the transcriptional response of cells of the same yeast strain, cultivated under different nitrogen availability conditions similar to those examined in our study, showed a remarkable upregulation of HXT3 at later fermentation stages presumably associated to the response to high ethanol concentrations [26,42]. Results from our study suggest that Hxt3p expression is not necessarily abrogated by glucose, or by nitrogen limitation, playing a major role throughout both fermentations, independently of the initial conditions tested. Specifically during the period of growth latency that followed ammonium supplementation, the glucose uptake kinetics revealed that the affinity for glucose remained unchanged and characteristic of a low-affinity glucose transporter such as Hxt3p, but that the transport capacity increased, suggesting that new glucose transporters had been synthesized. Although the results from the biclustering analysis indicated an increase in the transcription level of the moderate/high-affinity glucose transporter encoding gene HXT2 during the lag phase registered after ammonium refed, an affinity constant characteristic of Hxt2p could not be detected during this period. Following this period, the genes HXT3, HXT4, HXT6 and HXT7 coding for hexose transporters with distinct affinities for glucose were found to be transcriptionally activated, but kinetic evidences were only obtained for the presence of Hxt3p and Hxt6/7p. Although the activity of glucose transporters encoding genes is highly regulated by glucose concentration, the suggested derepression of glucose-repressible genes under the experimental conditions tested in this study can be associated to the decrease in MIG1 and RGT1 transcription level registered following ammonium refed. Also, the fact that plasma membrane $\mathrm{H}^{+}$-ATPase activity is activated by ethanol to counteract ethanol toxicity $[43,44]$, and that ethanol concentration significantly increase in RF broth after ammonium supplementation, the expression of different glucose uptake systems at this phase is a way to increase glycolytic flux and ATP production to support the requirements of an active plasma membrane $\mathrm{H}^{+}$-ATPase. It is known that ethanol leads to the transcriptional activation of HXT6 and HXT7 [45,46]. In the analysis of the results of glucose uptake kinetics we should have missed the identification of transporters with intermediate affinities such as those corresponding to the moderate/high-affinity glucose transporters Hxt4p and Hxt2p and of the moderate-affinity glucose transporter Hxt5p. Moreover, further research is needed to establish the presence and activity of Hxt2p and Hxt4p following ammonium supplementation. Nevertheless, the genes HXT1-HXT7 are, among a family of eighteen hexose permeases encoding genes, those that exhibit the highest transcript levels throughout the three fermentation conditions tested in our study [26].

\section{Conclusions}

There is a generalized idea that the main reason for stuck and sluggish alcoholic fermentations under nitrogen-limited conditions is the inhibition of sugar uptake as the result of the strong reduction of the activity of sugar transporters, triggered by protein synthesis arrest and transporters degradation. Although the glucose uptake capacity of yeast cells does indeed suffer a reduction during nitrogen starvation (about $80 \%$ of initial capacity), the glucose uptake was not eliminated and it was presumably maintained essentially by Hxt3p. Results also indicate that after ammonium supplementation, the recovery of the nitrogen-starved sluggish fermentation can be achieved by the increase of 
glucose uptake capacity, presumably related to the de novo synthesis of new transporters. This hypothesis was suggested by the transcription activation of HXT2 during the period of growth latency following ammonium supplementation and, subsequently, by the co-expression of Hxt3p, Hxt4p, Hxt6p and Hxt7p encoding genes, accompanying active fermentation recovery. Results of the glucose uptake kinetics also suggest the presence of glucose transporters with different glucose affinities and capacities, such as those exhibited by Hxt3p and Hxt6/7p. Results from this work provide new insights into the kinetics of glucose uptake in yeast cells throughout different fermentations, suggesting the combined effect of the presence of different nitrogen and glucose concentrations on the expression of HXT transcripts. For this reason, this study is useful to guide the development of more rational strategies to improve yeast performance when exposed to nutrient fluctuations and/or stressful conditions during alcoholic fermentation.

\section{Additional file}

Additional file 1: CCC-Biclusters of the transcriptional changes occurring following ammonium supplementation (RF) to the nitrogen-limited fermentation (LF). Two incubation time periods were compared following $80 \mathrm{~h}$ of LF fermentation (a control time point): i) after $8 \mathrm{~h}$ of incubation following ammonium supplementation (period 1) and ii) after a period of incubation extending from 8 to $24 \mathrm{~h}$ following ammonium supplementation (period 2). The transcriptional alterations during the selected periods are shown under parenthesis as $U$ (Upregulation), D (Downregulation) and N (No changes registered).

\section{Competing interests}

The authors declare that they have no competing interests.

\section{Authors' contributions}

MP performed and monitored alcoholic fermentations, glucose uptake assays and contributed to the analysis of the results and the writing of the manuscript under the scientific supervision of IS-C who conceived and coordinated the study. SCM performed the CCC-biclustering analysis. AM-F participated in the design of fermentation experiments and in discussions. All authors read and approved the final manuscript.

\section{Acknowledgements}

This research was supported by Fundação para a Ciência e a Tecnologia (FCT) (Contracts: ERA-IB/0002/2010 and post-doctoral fellowship [SFRH/BPD/ 73306/2010] awarded to MP). The authors thank Dr. J. Coutinho for the determination of ammonium concentrations and Prof. A. Mendes-Faia for encouragement and support.

\footnotetext{
Author details

${ }^{1}$ Institute for Biotechnology and Bioengineering, Centre for Biological and Chemical Engineering, Instituto Superior Técnico, Technical University of Lisbon, 1049-001 Lisbon, Portugal. ²Department of Bioengineering, Instituto Superior Técnico, Technical University of Lisbon, 1049-001 Lisbon, Portugal. ${ }^{3}$ Knowledge Discovery and Bioinformatics (KDBIO) Group, INESC-ID, Lisbon, Portugal. ${ }^{4}$ Department of Computer Science and Engineering, Instituto Superior Técnico, Technical University of Lisbon, 1049-001 Lisboa, Portugal. ${ }^{5}$ Institute for Biotechnology and Bioengineering, Centro de Genómica e Biotecnologia, Universidade de Trás-os-Montes e Alto Douro, Vila Real, Portugal.
}

Received: 30 April 2012 Accepted: 18 July 2012

Published: 30 July 2012

\section{References}

1. Graves T, Narendranath NV, Dawson K, Power R: Effect of pH and lactic or acetic acid on ethanol productivity by Saccharomyces cerevisiae in corn mash. J Ind Microbiol Biotechnol 2006, 33:469-474.

2. Garay-Arroyo A, Covarrubias AA, Clark I, Niño I, Gosset G, Martinez A: Response to different environmental stress conditions of industrial and laboratory Saccharomyces cerevisiae strains. Appl Microbiol Biotechnol 2004, 63:734-741.

3. Jackson RS: Wine Science. Principles and applications. 3rd edition. San Diego: Academic Press; 2008.

4. Teixeira MC, Mira NP, Sá-Correia I: A genome-wide perspective on the response and tolerance to food-relevant stresses in Saccharomyces cerevisiae. Curr Opin Biotechnol 2011, 22:150-156.

5. Jeronimo EM, Oliveira ES, Rocha Souza EL, Silva MA, Serra GE: Addition of proteic nitrogen during alcoholic fermentation for the production of cachaça. Sci Agric 2008, 65:161-168.

6. O'Connor-Cox ESC, Paik J, Ingeldew WM: Improved ethanol yields through supplementation with excess assimilable nitrogen. J Ind Microbiol 1991, 8:45-52.

7. Thomas KC, Ingledew WM: Fuel alcohol production: effects of free amino nitrogen on fermentation of very-high-gravity wheat mashes. Appl Environ Microbiol 1990, 56:2046-2050.

8. Bely M, Salmon JM, Barre P: Assimilable nitrogen addition and hexose transport system activity during enological fermentation. J Inst Brew 1994, 100:279-282.

9. Mauricio JC, Salmon JM: Apparent loss of sugar transport activity in Saccharomyces cerevisiae mainly account for maximum ethanol production during alcoholic fermentation. Biotechnol Lett 1992, 14:577-582.

10. Salmon JM: Effect of sugar transport inactivation in Saccharomyces cerevisiae on sluggish and stuck enological fermentations. Appl Environ Microbiol 1989, 55:953-958.

11. Salmon JM, Vincent O, Mauricio JC, Bely M, Barre P: Sugar transport inhibition and apparent loss of activity in Saccharomyces cerevisiae as a major limiting factor of enological fermentation. Am J Enol Vitic 1993, 44:56-64.

12. Reifenberger E, Freidel K, Ciriacy M: Identification of novel HXT genes in Saccharomyces cerevisiae reveals the impact of individual hexose transporters on glycolytic flux. Mol Microbiol 1995, 16:157-167

13. Buziol S, Warth L, Magario I, Freund A, Siemann-Herzberg M, Reuss M: Dynamic response of the expression of hxt1, hxt5 and hxt7 transport proteins in Saccharomyces cerevisiae to perturbations in the extracellular glucose concentration. J Biotechnol 2008, 134:203-210.

14. Özcan S, Johnston M: Three different regulatory mechanisms enable yeast hexose transporter (HXT) genes to be induced by different levels of glucose. Mol Cell Biol 1995, 15:1564-1572.

15. Liang H, Gaber RF: A novel signal transduction pathway in Saccharomyces cerevisiae defined by Snf3-regulated expression of HXT6. Mol Biol Cell 1996, 7:1953-1966.

16. Ye L, Berden JA, van Dam K, Kruckeberg AL: Expression and activity of the Hxt7 high-affinity hexose transporter of Saccharomyces cerevisiae. Yeast 2001, 18:1257-1267.

17. Reifenberger $E$, Boles E, Ciriacy M: Kinetic characterization of individual hexose transporters of Saccharomyces cerevisiae and their relation to the triggering mechanisms of glucose repression. Eur J Biochem 1997, 245:324-333.

18. Diderich JA, Schepper M, van Hoek P, Luttik MA, van Dijken JP, Pronk JT, Klaassen P, Boelens HF, de Mattos MJ, van Dam K, Kruckeberg AL: Glucose uptake kinetics and transcription of HXT genes in chemostat cultures of Saccharomyces cerevisiae. J Biol Chem 1999, 274:15350-15359.

19. Diderich JA, Schuurmans JM, Van Gaalen MC, Kruckeberg AL, Van Dam K: Functional analysis of the hexose transporter homologue HXT5 in Saccharomyces cerevisiae. Yeast 2001, 18:1515-1524.

20. Verwaal R, Paalman JWG, Hogenkamp A, Verkleij AJ, Verrips CT, Boonstra J: HXT5 expression is determined by growth rates in Saccharomyces cerevisiae. Yeast 2002, 19:1029-1038. 
21. Buziol S, Becker J, Baumeister A, Jung S, Mauch K, Reuss M, Boles E: Determination of in vivo kinetics of the starvation-induced Hxt5 glucose transporter of Saccharomyces cerevisiae. FEMS Yeast Res 2002, 2:283-291.

22. Krampe S, Stamm O, Hollenberg CP, Boles E: Catabolite inactivation of the high-affinity hexose transporters Hxt6 and Hxt7 of Saccharomyces cerevisiae occurs in the vacuole after internalization by endocytosis. FEBS Lett 1998, 441:343-347

23. van Suylekom D, van Donselaar E, Blanchetot C, Do Ngoc LN, Humbel BM, Boonstra J: Degradation of the hexose transporter Hxt5p in Saccharomyces cerevisiae. Biol Cell 2007, 99:13-23.

24. Kruckeberg AL, Ye L, Berden JA, van Dam K: Functional expression, quantification and cellular localization of the Hxt2 hexose transporter of Saccharomyces cerevisiae tagged with the green fluorescent protein. Biochem J 1999, 339:299-307.

25. Krampe S, Boles E: Starvation-induced degradation of yeast hexose transporter Hxt7p is dependent on endocytosis, autophagy and the terminal sequences of the permease. FEBS Lett 2002, 513:193-196.

26. Mendes-Ferreira A, del Olmo M, Garcia-Martinez J, Jimenez-Marti E, Mendes-Faia A, Perez-Ortin JE, Leão C: Transcriptional response of Saccharomyces cerevisiae to different nitrogen concentrations during alcoholic fermentation. App/ Environ Microbiol 2007, 73:3049-3060.

27. Madeira SC, Teixeira MC, Sa-Correia I, Oliveira AL: Identification of regulatory modules in time series gene expression data using a linear time biclustering algorithm. Trans Comput Biol Bioinform 2010, 7:153-165.

28. Mendes-Ferreira A, Mendes-Faia A, Leão C: Growth and fermentation patterns of Saccharomyces cerevisiae under different ammonium concentrations and its implications in winemaking industry. J Appl Microbiol 2004, 97:540-545

29. dos Santos SC, Tenreiro S, Palma M, Becker J, Sá-Correia I: Transcriptomic profiling of the Saccharomyces cerevisiae response to quinine reveals a glucose limitation response attributable to drug-induced inhibition of glucose uptake. Antimicrob Agents Chemother 2009, 53:5213-5223.

30. Walsh MC, Smits HP, Scholte M, van Dam K: Affinity of glucose transport in Saccharomyces cerevisiae is modulated during growth on glucose. J Bacteriol 1994, 176:953-958.

31. Coons DM, Boulton RB, Bisson LF: Computer-assisted nonlinear regression analysis of the multicomponent glucose uptake kinetics of Saccharomyces cerevisiae. J Bacteriol 1995, 177:3251-3258.

32. Maier A, Volker B, Boles E, Fuhrmann GF: Characterisation of glucose transport in Saccharomyces cerevisiae with plasma membrane vesicles (countertransport) and intact cells (initial uptake) with single Hxt1, Hxt2, Hxt3, Hxt4, Hxt6, Hxt7 or Gal2 transporters. FEMS Yeast Res 2002, 2:539-550.

33. Perez M, Luyten K, Michel R, Riou C, Blondin B: Analysis of Saccharomyces cerevisiae hexose carrier expression during wine fermentation: both lowand high-affinity Hxt transporters are expressed. FEMS Yeast Res 2005, 5:351-361.

34. Teixeira MC, Monteiro $P$, Jain $P$, Tenreiro S, Fernandes AR, Mira NP, Alenquer M, Freitas AT, Oliveira AL, Sá-Correia I: The YEASTRACT database: a tool for the analysis of transcription regulatory associations in Saccharomyces cerevisiae. Nucleic Acids Res 2006, 34:D446-D451.

35. Georis I, Feller A, Vierendeels F, Dubois E: The yeast GATA factor Gat1 occupies a central position in nitrogen catabolite repression-sensitive gene activation. Mol Cell Biol 2009, 29:3803-3815.

36. Zhang N, Wu J, Oliver SG: Gis1 is required for transcriptional reprogramming of carbon metabolism and the stress response during transition into stationary phase in yeast. Microbiology 2009, 155:1690-1698.

37. Lai LC, Kosorukoff AL, Burke PV, Kwast KE: Dynamical remodeling of the transcriptome during short-term anaerobiosis in Saccharomyces cerevisiae: differential response and role of Msn2 and/or Msn4 and other factors in galactose and glucose media. Mol Cell Biol 2005 25:4075-4091

38. Verwaal $R$, Arako M, Kapur R, Verkleij AJ, Verrips CT, Boonstra J: HXT5 expression is under control of STRE and HAP elements in the HXT5 promoter. Yeast 2004, 21:747-757.

39. Tomas-Cobos L, Casadome L, Mas G, Sanz P, Posas F: Expression of the HXT1 low affinity glucose transporter requires the coordinated activities of the HOG and glucose signalling pathways. J Biol Chem 2004, 279:22010-22019.
40. Tomas-Cobos L, Viana R, Sanz P: TOR kinase pathway and 14-3-3 proteins regulate glucose-induced expression of $H X T 1$, a yeast low-affinity glucose transporter. Yeast 2005, 22:471-479.

41. Schmelzle T, Beck T, Martin DE, Hall MN: Activation of the RAS/cyclic AMP pathway suppresses a TOR deficiency in yeast. Mol Cell Biol 2004, 24:338-351.

42. Karpel JE, Place WR, Bisson LF: Analysis of the Major Hexose Transporter Genes in Wine Strains of Saccharomyces cerevisiae. Am J Enol Vitic 2008, 59:265-275.

43. Monteiro GA, Sá-Correia I: In vivo activation of yeast plasma membrane $\mathrm{H}^{+}$-ATPase by ethanol: effect on the kinetic parameters and involvement of the carboxyl-terminus regulatory domain. Biochim Biophys Acta 1998, 1370:310-316.

44. Rosa MF, Sá-Correia I: In vivo activation by ethanol of plasma membrane ATPase of Saccharomyces cerevisiae. Appl Environ Microbiol 1991, 57:830-835.

45. Gasch AP, Spellman PT, Kao CM, Carmel-Harel O, Eisen MB, Storz G, Botstein D, Brown PO: Genomic expression programs in the response of yeast cells to environmental changes. Mol Biol Cell 2000, 11:4241-4257.

46. Marks VD, Ho Sui SJ, Erasmus D, van der Merwe GK, Brumm J, Wasserman WW, Bryan J, van Vuuren HJ: Dynamics of the yeast transcriptome during wine fermentation reveals a novel fermentation stress response. FEMS Yeast Res 2008, 8:35-52.

doi:10.1186/1475-2859-11-99

Cite this article as: Palma et al:: Impact of assimilable nitrogen

availability in glucose uptake kinetics in Saccharomyces cerevisiae during alcoholic fermentation. Microbial Cell Factories 2012 11:99.

\section{Submit your next manuscript to BioMed Central and take full advantage of:}

- Convenient online submission

- Thorough peer review

- No space constraints or color figure charges

- Immediate publication on acceptance

- Inclusion in PubMed, CAS, Scopus and Google Scholar

- Research which is freely available for redistribution 